\title{
A RIESZ REPRESENTATION THEOREM IN THE SETTING OF LOCALLY CONVEX SPACES $\left({ }^{1}\right)$
}

\author{
BY \\ ROBERT K. GOODRICH
}

Introduction. Let $H$ be a compact Hausdorff space, let $E$ and $F$ be locally convex topological vector spaces over the real or complex field where $F$ is Hausdorff. Let $C(H, E)$ be the space of continuous functions from $H$ into $E$ with the topology of uniform convergence. The general problem is to find an integral representation theorem for a continuous linear transformation $T$ from $C(H, E)$ into $F$.

The well-known Riesz representation theorem [3] gives a Stieltjes integral representation for $T$ when $H$ is a closed interval and $E$ and $F$ are the real numbers. Tucker [6] gives a Stieltjes' integral type representation for $T$ in the case of $H$, a closed interval, and $E$ and $F$, linear normed spaces. This paper was generalized by Uherka [7] to the case of $H$, a compact space, and $E$ and $F$, linear normed spaces. Also see Swong's paper [5] in which $H$ is a compact Hausdorff space and $E$ and $F$ are locally convex topological vector spaces. This last paper takes a fundamentally different approach from that of Tucker and Uherka, and Swong writes $y^{\prime} T$ as an integral where $y^{\prime}$ is in the topological dual $F^{\prime}$ of $F$. However, Swong is able to write $T$ as an integral under various additional assumptions on $E, F$ or $T$. The approach taken in this paper more closely follows that of Tucker and Uherka where a much smaller class of sets is employed in the definition of integral. The result is that the integral converges in the $\varepsilon^{00}$ topology, as compared to the weak topology in Swong's paper. And $T$ is written as an integral.

1. Notation and preliminaries. Let $C(H, E)$ be the continuous functions from $H$ into $E$, where the topology for $C(H, E)$ is generated by the seminorms $\{p\}$ where $p(f)=\sup _{t \in H} p(f(t))$ as $p$ ranges over the continuous seminorms on $E$. Let $L(E, F)$ be the space of continuous linear transformations of $E$ into $F$, with the topology given by the seminorms $\left\{q^{M}\right\}$, where for each continuous seminorm $q$ on $F$ and each bounded subset $M$ of $E$ and each $u$ in $L(E, F), q^{M}(u)=\sup _{x \in M} q(u(x))$. Let $C(H)$ be the continuous functions from $H$ into our field, which is either the complex or real number field, with the usual supremum norm. We denote this norm $\|f\|$ for $f$ in $C(H)$. If $q$ is any continuous seminorm on $F$ let the " $q$-unit ball" be denoted by $B_{q}$, i.e. the set of all $y$ in $E$ such that $q(y) \leqq 1$, and let $B_{q}^{O}$ be the polar of $B_{q}$, i.e. the set of all $y^{\prime}$ in $F^{\prime}$, such that $\left|\left\langle y, y^{\prime}\right\rangle\right| \leqq 1$ for every $y$ in $B_{q}$.

Presented to the Society, November 14, 1966; received by the editors January 13, 1967.

(1) This paper is a substantial part of the author's doctoral dissertation submitted to the University of Utah in August, 1966. The author wishes to thank Dr. Don H. Tucker, his thesis advisor, for his assistance. 
Suppose $G$ is a locally convex topological space which is also Hausdorff. Let $G^{\prime}$ be the topological dual of $G$ under the $\beta\left(G^{\prime}, G\right)$ topology, i.e. the topology of uniform convergence on weakly bounded subsets of $G$, which is the polar topology generated by the weakly bounded subsets of $G$. Let $G^{\prime \prime}$ be the dual of $G^{\prime}$ under the $\varepsilon^{00}$ topology, i.e. the topology of uniform convergence on equicontinuous subsets of $G^{\prime}$. (See $[4$, p. 71$]$.) We note that the $\varepsilon^{00}$ topology is generated by the seminorms

$$
q^{+}\left(g^{\prime \prime}\right)=\sup _{g^{\prime} \in B_{q}^{O}}\left|\left\langle g^{\prime \prime}, g^{\prime}\right\rangle\right|
$$

and that if $g \in G, q^{+}(g)=q(g)$. The weak topology $\sigma\left(G^{\prime}, G\right)$ on $G^{\prime}$ is the topology of uniform convergence on finite subsets of $G$. These various topologies are discussed in [4].

Both Tucker [6] and Uherka [7] made use of what they called the weak sequential extension of $G$, denoted by $G^{+} . G^{+}$is the set of all $g^{\prime \prime}$ in $G^{\prime \prime}$ such that there exists a sequence $\left\{g_{n}\right\}$ in $G$ such that $\left\langle g^{\prime \prime}, g^{\prime}\right\rangle=\lim \left\langle g_{n}, g^{\prime}\right\rangle$ for every $g^{\prime}$ in $G^{\prime}$. We denote this by $g^{\prime \prime} \sim\left\{g_{n}\right\}$. We note that $G^{+}$is a linear subspace of $G^{\prime \prime}$, and we take the topology induced by $\varepsilon^{00}$ for the topology of $G^{+}$.

We shall also make use of some notation used in Swong's paper [5]. When we say "there exists a pairing $(p, q)$ such that ..." we shall mean "for each such continuous seminorm $q$ on $F$ there exists a continuous seminorm $p$ on $E$ such that ...". For example, a linear transformation $u$ from $E$ to $F$ is continuous if and only if there exists a pairing $(p, q)$ such that $q$ is bounded on $u\left(B_{p}\right)$. Also, $T$ is continuous if and only if there exists a pairing $(p, q)$ such that

$$
|T|_{p-q}=\sup \{q T(f), p(f) \leqq 1\}
$$

exists where $p(f)=\sup _{t \in H} p f(t)$, and whenever $|T|_{p-q}$ exists, we say $T$ is $p-q$ related.

We say that $y^{\prime} \in G^{\prime}$ is $q$-related when $\left|y^{\prime}\right|_{q}=\sup _{y \in B_{q}}\left|\left\langle y, y^{\prime}\right\rangle\right|$ exists.

2. The mapping $J$. Recall that $T$ is a continuous linear transformation of $C(H, E)$ into $F$. We shall define the mapping $J$ by $J(f)(x)=T(f \cdot x)$ where $f$ is in $C(H)$ and $x$ is in $E$, and $(f \cdot x)(t)=f(t) x$ for every $t$ in $H$. We notice that $J$ maps $C(H)$ continuously into $L(E, F)$. The main purpose of this section is to, in some sense, extend $J$ to operate on the characteristic functions of a certain class of subsets $R$ of $H$. This will be accomplished by noting that the bitranspose $J^{\prime \prime}$ of $J$ maps $C^{+}(H)$ into $L^{+}(E, F)$, and we shall show that the characteristic functions of sets in $R$ are imbedded in a natural way in $C^{+}(H)$. It will further be shown that there is a natural linear mapping $\theta$ of $L^{+}(E, F)$ into $\Gamma\left(E, F^{+}\right)$, the class of linear transformation of $E$ into $F^{+}$. A set function $K$ will be defined on $R$ by $K(e)=\theta J^{\prime \prime}\left(\chi_{e}^{\prime \prime}\right)$ where $\chi_{e}$ is the characteristic function of $e$ and $e$ is in $R$. It is with respect to this set function $K$ that we will define our integral. The proofs of the propositions and lemmas in this section will be omitted as they are either immediate or similar to those in [6]. 
Proposition 2.1. $J$ is a continuous linear transformation of $C(H)$ into $L(E, F)$.

Proposition 2.2. The bitranspose $J^{\prime \prime}$ of $J$ maps $C^{+}(H)$ into $L^{+}(E, F)$, and in fact if $f^{\prime \prime} \sim\left\{f_{n}\right\}$, then $J^{\prime \prime}\left(f^{\prime \prime}\right) \sim\left\{J\left(f_{n}\right)\right\}$.

Definition 2.1. We say the sequence $\left\{x_{n}\right\}$ in the locally convex topological Hausdorff space $G$ converges weakly if $\lim \left\langle x_{n}, x^{\prime}\right\rangle$ exists for every $x^{\prime}$ in $G^{\prime}$.

LeMma 2.1. If $\left\{x_{n}\right\}$ converges weakly then there exists a unique $x^{\prime \prime}$ in $G^{+}$such that $x^{\prime \prime} \sim\left\{x_{n}\right\}$.

Proposition 2.3. There exists a linear mapping $\theta$ of $L^{+}(E, F)$ into $\Gamma\left(E, F^{+}\right)$ such that if $u^{\prime \prime} \sim\left\{u_{n}\right\}$ then $\theta\left(u^{\prime \prime}\right)(x) \sim\left\{u_{n}(x)\right\}$.

Definition 2.2. $R$ is the collection of subsets $e$ of $H$ such that there exists a sequence $\phi_{n}=f_{n}-g_{n}$ where: (1) $f_{n}$ and $g_{n}$ are in $C(H)$, (2) $f_{n}$ and $g_{n}$ are nonnegative and nonincreasing in $n$, and (3) $\phi_{n}(t)$ converges to $\chi_{e}(t)$ for each $t$ in $H$ where $\chi_{e}$ is the characteristic function of $e$, i.e. $\chi_{e}(t)$ is zero for $t$ not in $e$ and is one otherwise.

Uherka [7, Lemma 6.1, p. 21] shows $R$ is a ring, i.e. $R$ is closed under finite unions, finite intersections, and differences. Also $\Phi$ (the empty set) and $H$ are in $R$. Also if $e$ is a closed $G_{\delta}$, i.e. a closed set that is the intersection of a countable number of open sets, or if $e$ is the difference of two closed $G_{\delta}$ 's, then $e$ is in $R$. By [7, Theorem 3.3, p. 10], if $e$ is in $R$ and $\phi_{n}$ and $\phi_{n}^{\prime}$ are any two sequences satisfying the conditions of Definition 2.2 above, then $\lim \left\langle\phi_{n}, f^{\prime}\right\rangle, \lim \left\langle\phi_{n}^{\prime}, f^{\prime}\right\rangle$ exist and are equal for every $f^{\prime}$ in $C^{\prime}(H)$. But then by Lemma 2.1 above, there exists an element $\chi_{e}^{\prime \prime}$ of $C^{+}(H)$ such that $\chi_{e}^{\prime \prime} \sim\left\{\phi_{n}\right\}$. We see also that $\chi_{e}^{\prime \prime} \sim\left\{\phi_{n}^{\prime}\right\}$ for any sequence $\left\{\phi_{n}^{\prime}\right\}$ satisfying the conditions of Definition 2.2 .

Definition 2.3. The set function $K$ is defined on $R$ by $K(e)=\theta \cdot J^{\prime \prime}\left(\chi_{e}^{\prime \prime}\right)$.

Thus we see $K$ maps $R$ into $\Gamma\left(E, F^{+}\right)$.

LEMMA 2.2. $K$ is finitely additive.

3. Partitions and the set function $K$. In this section we shall develop some properties of our set function $K$, and we shall define several notions of partition which will be used in defining our integral and in proving the representation theorem. The various definitions of partition are the same as Uherka [7] defined them.

Definition 3.1. If $e_{i}$ is in $R$ for $i=1, \ldots, n$ where the $e_{i}$ are disjoint, and if $e_{i} \cup \ldots \cup e_{n}=H$, then we call $P=\left\{e_{1}, e_{2}, \ldots, e_{n}\right\}$ a partition of $H$.

Definition 3.2. Suppose $P=\left\{e_{1}, \ldots, e_{n}\right\}$ is a partition of $H$, and $p$ is a continuous seminorm on $E$. Then $P$ is an $\varepsilon-p$ partition of $H$ with respect to $f$ means that for each $i(1 \leqq i \leqq n)$ and for each pair $x, x^{\prime}$ in $f\left(e_{i}\right), p\left(x-x^{\prime}\right) \leqq \varepsilon$.

Definition 3.3. The partition $P=\left\{e_{1}, \ldots, e_{n}\right\}$ is called an essential partition of $H$ if and only if $e_{1} \cup \ldots \cup e_{i}$ is a closed $G_{\delta}$ for $i=1, \ldots, n$, and each $e_{i}$ is nonempty.

Definition 3.4. If the essential partition $P$ is also an $\varepsilon-p$ partition of $H$ with respect to $f$ then we say $P$ is an $\varepsilon-p$ essential partition of $H$ with respect to $f$. 
Definition 3.5. If $U=\left\{U_{1}, \ldots, U_{n}\right\}$ is a finite open covering of $H$, then a set of functions $\left\{\phi_{1}, \ldots, \phi_{n}\right\}$ is called a partition of unity subordinate to $U$ if for $i=1, \ldots, n$ (1) $\phi_{i} \in C(H),(2) 0 \leqq \phi_{i} \leqq 1,(3) \phi_{i}(t)=0$ for $t$ not in $U_{i}$, and (4) $\phi_{i}(t)+\cdots+\phi_{n}(t)=1$ for every $t$ in $H$.

The following lemma is proved in [7, Theorem 7.1, p. 28].

LEMMA 3.1. If $P=\left\{e_{1}, e_{2}, \ldots, e_{n}\right\}$ is an essential partition of $H$, then there exists a sequence $\left\{U_{k}\right\}=\left\{U_{k 1}, \ldots, U_{k n}\right\}$ of open coverings of $H$ and a sequence $\left\{\phi_{k 1}, \ldots, \phi_{k n}\right\}$ where $\left\{\phi_{k 1}, \ldots, \phi_{k n}\right\}$ is a partition of unity subordinate to $U_{k}(k=1,2, \ldots)$, and (1) $e_{1} \cup \ldots \cup e_{i} \subset U_{k i}, i=1, \ldots, n, k=1,2, \ldots$, (2) for $i=1, \ldots, n,\left\{\phi_{k i}\right\}$ is the difference of two nonincreasing, nonnegative sequences in $C(H)$, (3) for each $i$, $\lim _{k} \phi_{k i}(t)=\chi_{e_{i}}(t)$ for all $t$ in $H$.

LEMMA 3.2. If $f \in C(H, E)$ and $\varepsilon>0$ and $p$ is a continuous seminorm on $E$, then there exists an $\varepsilon-p$ essential partition of $H$ with respect to $f$.

Proof. Since $H$ is compact and $f$ is continuous $f(H)$ is compact. So $f(H)$ can be covered with a finite collection $U_{1}, U_{2}, \ldots, U_{n}$ where $U_{i}=\left\{x: p\left(x-x_{i}\right) \leqq \varepsilon / 2\right.$, $\left.x_{i} \in f(H)\right\}$. Without loss of generality we can assume that no $U_{i} \cap f(H)$ is contained in the union of the remaining $U_{i}$ 's, for this can be achieved by taking a subcollection of $U_{i}, \ldots, U_{n}$. Each $U_{i}$ is a closed $G_{\delta}$, for $U_{i}$ is closed, and $U_{i}=\bigcap C_{n}$ where $C_{n}=\left\{x: p\left(x-x_{i}\right)<\varepsilon / 2+1 / n\right\}$, and each $C_{n}$ is open. Let $A_{i}=f^{-1}\left(U_{i}\right) . f$ is continuous and $U_{i}$ is closed, so $A_{i}$ is closed. Also $A_{i}=f^{-1}\left(U_{i}\right)=f^{-1}\left(\cap C_{n}\right)$ $=\bigcap f^{-1}\left(C_{n}\right)$ and each $f^{-1}\left(C_{n}\right)$ is open, so $A_{i}$ is a closed $G_{\delta}$. Now let $e_{1}=A_{1}$, $e_{i}=A_{i}-\left(A_{1} \cup \cdots \cup A_{i-1}\right), i=2, \ldots, n . e_{i} \cup \cdots \cup e_{i}=A_{1} \cup \cdots \cup A_{i}$ which is the finite union of closed $G_{\delta}$ 's, and hence is a closed $G_{\delta}$. Checking the other conditions we see $P=\left\{e_{1}, \ldots, e_{n}\right\}$ is an $\varepsilon-p$ essential partition of $H$ with respect to $f$.

Definition 3.6. If $W$ is a finitely additive set function from $R$ into $\Gamma\left(E, F^{+}\right)$, then we say $W$ is of bounded $\left(p, q^{+}\right)$variation if there exists a pairing $(p, q)$ and $W_{p-q^{+}} \geqq 0$ such that if $P=\left\{e_{1}, \ldots, e_{n}\right\}$ is any essential partition and $x_{1}, \ldots, x_{n}$ are in $E$ then $q^{+}\left(\sum W\left(e_{i}\right) \cdot x_{i}\right) \leqq W_{p-q^{+}} \max p\left(x_{i}\right)$. Denote by $V(W)_{p-q^{+}}$the greatest lower bound of all such $W_{p-q^{+}}$.

Proposition 3.1. The set function $K$ is of bounded $\left(p-q^{+}\right)$-variation, and for each pair $(p, q)$ such that $T$ is $p-q$ related, we have that $V(K)_{p-q^{+}} \leqq|T|_{p-q}$.

Proof. Suppose $P=\left\{e_{1}, \ldots, e_{n}\right\}$ is an essential partition of $H$. From Lemma 3.1, there exists a sequence $\left\{\phi_{k 1}, \ldots, \phi_{k n}\right\}$ of partitions of unity subordinate to some open coverings $U_{k}$ for each $k$ such that $\lim _{k} \phi_{k i}(t)=\chi_{e_{i}}(t)$ for every $t$, and each $\left\{\phi_{k i}\right\}$ is the difference of sequences satisfying the conditions in Definition 2.2, therefore $\chi_{e_{i}}^{\prime \prime} \sim\left\{\phi_{k i}\right\}$. Thus $q^{+}\left(\sum K\left(e_{i}\right) \cdot x_{i}\right)=q^{+}\left(\sum \theta J^{\prime \prime}\left(\chi_{e_{i}}^{\prime \prime}\right) \cdot x_{i}\right)$. Since $\chi_{e_{i}}^{\prime \prime} \sim\left\{\phi_{k i}\right\}$, then $J^{\prime \prime}\left(\chi_{e_{i}}^{\prime \prime}\right) \sim\left\{J\left(\phi_{k i}\right)\right\}$ by Proposition 2.2. Also $\theta J^{\prime \prime}\left(\chi_{e_{i}}^{\prime \prime}\right) \cdot x_{i} \sim\left\{J\left(\phi_{k i}\right) \cdot x_{i}\right\}$ by Proposition 2.3. But $J\left(\phi_{k i}\right) \cdot x_{i}=T\left(\phi_{k i} x_{i}\right)$ by definition. Thus

$$
q^{+}\left(\sum \theta J^{\prime \prime}\left(\chi_{e_{i}}^{\prime \prime}\right) \cdot x_{i}\right)=\sup \left\{\lim _{k}\left|\left\langle\sum_{i} T\left(\phi_{k i} \cdot x_{i}\right), y^{\prime}\right\rangle\right|, y^{\prime} \in B_{q}^{o}\right\} .
$$


But

$$
\sup \left\{\left|\left\langle T(f), y^{\prime}\right\rangle\right|, y^{\prime} \in B_{q}^{O}\right\}=q T(f) \leqq|T|_{p-q} p(f)
$$

for some continuous seminorm $p$ on $E$. Thus $\left|\left\langle T(f), y^{\prime}\right\rangle\right| \leqq|T|_{p-q} p(f)$ for every $y^{\prime} \in B_{q}^{O}$. So

$$
\left|\left\langle\sum_{i} T\left(\phi_{k i} \cdot x_{i}\right), y^{\prime}\right\rangle\right|=\left|\left\langle T\left(\sum_{i} \phi_{k i} \cdot x_{i}\right), y^{\prime}\right\rangle\right| \leqq|T|_{p-q} p\left(\sum_{i} \phi_{k i} \cdot x_{i}\right)
$$

for every $y^{\prime} \in B_{q}^{O}$ but

$$
\begin{aligned}
|T|_{p-q} p\left(\sum_{i} \phi_{k i} \cdot x_{i}\right) & =|T|_{p-q} \max _{t \in H} p\left(\sum_{i} \phi_{k i}(t) \cdot x_{i}\right) \\
& \leqq|T|_{p-q} \max _{t \in H} \sum_{i} \phi_{k i}(t) p\left(x_{i}\right) \\
& \leqq|T|_{p-q}\left(\max _{t \in H} \sum_{i} \phi_{k i}(t)\right) \max p\left(x_{i}\right) .
\end{aligned}
$$

But $\sum_{i} \phi_{k i}(t)=1$ for every $t \in H$. Thus $q^{+}\left(\sum K\left(e_{i}\right) \cdot x_{i}\right) \leqq|T|_{p-q} \max p\left(x_{i}\right)$. Thus $K$ is of bounded $p-q^{+}$variation, and $V(K)_{p-q^{+}} \leqq|T|_{p-q}$. We have the following corollary:

COROLlARY 3.1. If $e=e_{1}-e_{2}$ where $e_{1}$ and $e_{2}$ are closed $G_{\delta}^{\prime}$ 's, then $K(e) \in L\left(E, F^{+}\right)$; and if $T$ is $p-q$ related, $K(e)$ is $p-q^{+}$related and $|K(e)|_{p-q^{+}} \leqq|T|_{p-q}$ where $|K(e)|_{p-q^{+}}$is the $p-q^{+}$norm of $K(e)$ in $L\left(E, F^{+}\right)$.

DEFINITION 3.7. If $f$ is a mapping of $H$ into $E$ and $W$ is an additive set function of $R$ into $\Gamma\left(E, F^{+}\right)$, then we say $f$ is integrable with respect to $W$ if the following holds: there exists a $y^{\prime \prime} \in F^{\prime \prime}$ such that if $q^{+}$is given, where $q$ is any continuous seminorm on $F$, and $\varepsilon>0$ is given, then there exists an essential partition $P_{0}$ such that if $P=\left\{e_{1}, \ldots, e_{n}\right\}$ is any essential partition which is a refinement of $P_{0}$, then $q^{+}\left(y^{\prime \prime}-\sum_{i=1}^{n} W\left(e_{i}\right) \cdot x_{i}\right)<\varepsilon$ for any choice $x_{i} \in f\left(e_{i}\right)$. In this case we denote $y^{\prime \prime}$ by $\int d W f$.

We shall not only show that $\int d K f$ exists for every $f \in C(H, E)$ but that $T(f)$ $=\int d K(f)$. We shall also show that if $T$ is $p-q$ related, then $K$ is of bounded $p-q^{+}$variation and $|T|_{p-q}=V(K)_{p-q^{+}}$. The rest of this section develops lemmas leading to this result.

Proposition 3.2. Let $P_{e}=\left\{e_{1}, \ldots, e_{n}\right\}, P_{E}=\left\{E_{1}, \ldots, E_{n}\right\}$ be two $\varepsilon-p$ essential partitions of $H$ with respect to $f$, where $f$ maps $H$ into $E$. Suppose that $x_{i} \in f\left(e_{i}\right)$, $i=1, \ldots, n$, and $y_{i} \in f\left(E_{j}\right), j=1,2, \ldots, m$. Then, if $W$ is finitely additive and is of $p-q^{+}$bounded variation,

$$
q^{+}\left(\sum_{i=0}^{n} W\left(e_{i}\right) \cdot x_{i}-\sum_{j=1}^{m} W\left(E_{j}\right) \cdot y_{j}\right) \leqq V(W)_{p-q^{+}}(2 \varepsilon) .
$$


Proof. Let $S_{i j}=e_{i} \cap E_{j}$. Then $S_{11}, S_{21}, \ldots, S_{n 1}, \ldots, S_{12}, \ldots, S_{n 2}, \ldots, S_{1 m}, \ldots, S_{n m}$ is an essential partition of $H$ if we disregard any empty sets. But $e_{i}=\bigcup_{j=1}^{m}\left(e_{i} \cap E_{j}\right)$, so

$$
\begin{gathered}
W\left(e_{i}\right)=\sum_{j=1}^{m} W\left(e_{i} \cap E_{j}\right), \\
\sum_{i=1}^{n} W\left(e_{i}\right) \cdot x_{i}=\sum_{i=1}^{n} \sum_{j=1}^{m} W\left(e_{i} \cap E_{j}\right) \cdot x_{i},
\end{gathered}
$$

and similarly

$$
\sum_{j=1}^{m} W\left(E_{j}\right) \cdot y_{j}=\sum_{i=1}^{n} \sum_{i=1}^{m} W\left(e_{i} \cap E_{j}\right) \cdot y_{j} .
$$

(Here we have interchanged the order of summation.) Thus

$$
q^{+}\left[\sum_{i=1}^{n} W\left(e_{i}\right) \cdot x_{i}-\sum_{j=1}^{m} W\left(E_{j}\right) \cdot y_{j}\right]=q^{+}\left[\sum_{i=1}^{n} \sum_{j=1}^{m} W\left(S_{i j}\right)\left(x_{i}-y_{j}\right)\right] .
$$

But if $e_{i} \cap E_{j}=\varnothing$, then $W\left(S_{i j}\right)=0$; thus we may replace $x_{i}-y_{j}$ in such terms by zero. But if $e_{i} \cap E_{j}$ is not empty then $p\left(x_{i}-y_{j}\right) \leqq 2 \varepsilon$; thus since $W$ is of bounded $p-q^{+}$variation we have

$$
q^{+} \sum_{i=1}^{n} \sum_{j=1}^{m} W\left(S_{i j}\right)\left(x_{i}-y_{j}\right) \leqq V(W)_{p-q^{+}}(2 \varepsilon) .
$$

Proposition 3.3. Suppose $f \in C(H, E), \varepsilon>0$, and $p$, a continuous seminorm on $E$, are given, then there exists an $\varepsilon-p$ essential partition $P=\left\{e_{1}, \ldots, e_{n}\right\}$ of $H$ with respect to $f$ and a sequence of partitions of unity $\left\{\phi_{k 1}, \ldots, \phi_{k n}\right\}, k=1,2, \ldots$, subordinate to some open coverings $U_{k}$ of $H$ such that the following conditions are satisfied for each $i=1,2, \ldots, n$ : (1) the sequence $\left\{\phi_{k i}\right\}$ is nonnegative and is the difference of two nonincreasing, nonnegative sequences in $C(H)$, (2) for each $t \in H$, $\lim _{k} \phi_{k i}(t)=\chi_{e_{i}}(t)$, (3) $p\left(f-\sum_{i=1}^{n} \phi_{k i} \cdot y_{i}\right) \leqq 2 \varepsilon$ for all $k$ and for any choice of the points $y_{i} \in f\left(e_{i}\right)$.

Proof. Let $U_{1}, \ldots, U_{n}$ be the subsets of $E$ as defined in the proof of Lemma 3.2, and let $A_{i}=f^{-1}\left(U_{i}\right), e_{i}=A_{i}-\left(A_{i} \cup \cdots \cup A_{i-1}\right)$ for $2 \leqq i \leqq n$, and $e_{1}=A_{1}$. As we saw in the proof of Lemma 3.2, $A_{i}$ is a closed $G_{\delta}$. Let $O_{k i}=\bigcup_{x_{0} \in U_{t}}\left\{x: p\left(x-x_{0}\right)<\varepsilon / 2 k\right\}$ and let $U_{k i}=f^{-1}\left(O_{k i}\right)$. Then $U_{i}=\bigcap_{k} O_{k i}$. So

$$
A_{i}=f^{-1}\left(U_{i}\right)=f^{-1}\left(\bigcap_{k} O_{k i}\right)=\bigcap_{k} f^{-1}\left(O_{k i}\right)=\bigcap_{k} U_{k i} \text {. }
$$

For each $i$, there exists a nonincreasing sequence of functions $h_{k i}(k=1,2, \ldots)$ in $C(H)$ such that $0 \leqq h_{k i}(t) \leqq 1$ for all $t \in H$ and all $k, h_{k i}(t)=1$ if $t \in A_{i}, h_{k i}(t)=0$ for all $k$ if $t$ is not in $U_{k i}$, and $\lim _{k} h_{k i}(t)=\chi_{A_{i}}(t)$ for each $t \in H$. See [7, Lemma 6.1, p. 18]. Now let

$$
\begin{aligned}
& \phi_{k 1}=h_{k 1}, \quad \phi_{k 2}=h_{k 2}-\min \left(h_{k 2}, \phi_{k 1}\right), \\
& \phi_{k n}=h_{k n}-\min \left(h_{k n}, \phi_{k 1}+\cdots+\phi_{k, n-1}\right) .
\end{aligned}
$$


(1) $\phi_{k i}$ is the difference of the two nonnegative, nondecreasing sequences in $C(H)$, and $\phi_{k 1}+\cdots+\phi_{k i}$ is nonnegative and nonincreasing in $C(H)$ for $i=1, \ldots, n$.

(2) For each $i, \phi_{k i}(t)=0$ if $t$ is not in $U_{k i}(k=1,2, \ldots)$. (3) For each $i, \lim _{k} \phi_{k i}(t)$ $=\chi_{e_{i}}(t)$ for all $t$ in $H$. (4) $\phi_{k 1}(t)+\cdots+\phi_{k i}(t)=1$ for $t$ in $e_{1} \cup \cdots \cup e_{i}$ for $i=1, \ldots, n$.

The above statements follow easily from induction arguments.

Next suppose $t \in H$ and consider $p\left(f(t)-\sum_{i=1}^{n} \phi_{k i}(t) \cdot y_{i}\right)$, where $y_{i} \in f\left(e_{i}\right)$. Since $t \in H, t \in e_{j}$ for some $j$, and $e_{j} \subset A_{j} \subset U_{k j}$, so $f(t) \in f\left(U_{k j}\right)$. If $U_{k j} \cap U_{k i}$ is not empty, then $f\left(U_{k j}\right) \cap f\left(U_{k i}\right)$ is not empty. But $f\left(U_{k i}\right) \subset O_{k i}$ and diam $p\left(O_{k i}\right) \leqq 2 \varepsilon$. That is, if $x, x^{\prime} \in O_{k i}, p\left(x-x^{\prime}\right) \leqq 2 \varepsilon$ for $i=1, \ldots, n$. Suppose $t \in U_{k i}$, then $f(t) \in f\left(U_{k i}\right)$ and $x_{i} \in f\left(e_{i}\right) \subset f\left(U_{k i}\right)$, thus $p\left(f(t)-x_{i}\right) \leqq 2 \varepsilon$. Let $\Sigma^{\prime}$ denote the summation over all $i$ such that $t \in U_{k i}$. But if $t$ is not in $U_{k i}$, then $\phi_{k i}(t)=0$. Thus

$$
\begin{aligned}
p\left(f(t)-\sum_{i=1}^{n} \phi_{k i}(t) \cdot y_{i}\right) & =p\left(f(t)-\sum^{\prime} \phi_{k i}(t) \cdot y_{k}\right) \\
& =p\left(f(t) \sum^{\prime} \phi_{k i}(t)-\sum^{\prime} \phi_{k i}(t) y_{i}\right)
\end{aligned}
$$

because $\left\{\phi_{k 1}, \ldots, \phi_{k n}\right\}$ is a partition of unity subordinate to $\left\{U_{k 1}, \ldots, U_{k n}\right\}$, so $\sum^{\prime} \phi_{k i}(t)=1$. But

$$
p\left(\sum^{\prime} \phi_{k i}(t) f(t)-\sum^{\prime} \phi_{k i}(t) y_{i}\right) \leqq p\left(\sum^{\prime} \phi_{k i}(t) p\left(f(t)-y_{i}\right) \leqq \sum^{\prime} \phi_{k i}(t) 2 \varepsilon=2 \varepsilon .\right.
$$

Thus $p\left(f-\sum_{i=1}^{n} \phi_{k i} \cdot y_{i}\right) \leqq 2 \varepsilon$ for all $k$ and any choice of $y_{i} \in f\left(e_{i}\right)$.

4. The representation theorem. In this section we shall prove the representation theorem stated in the last section.

LEMMA 4.1. If $f \in C(H, E)$, then given $q^{+}$, where $q$ is a continuous seminorm on $F$, then there exists a continuous seminorm $p$ on $E$ such that if $d>0$ is given, then there exists a $\delta\left(d, q^{+}, p\right)$ such that if $0<\varepsilon \leqq \delta\left(d, q^{+}, p\right)$, then $q^{+}\left(T(f)-\sum_{i=1}^{n} K\left(e_{i}\right) \cdot x_{i}\right)<d$ for any $\varepsilon-p$ essential partition $P=\left\{e_{1}, \ldots, e_{n}\right\}$ and any choice $x_{i} \in f\left(e_{i}\right)$.

Proof. Suppose $q$ is given, then there exists a continuous seminorm $p$ on $E$ such that $T$ is $p-q$ related. Now pick a sequence $\varepsilon_{n}>0$ converging monotonically to zero. From Proposition 3.3 we know that for each $n$ there exists an $\varepsilon_{n}-p$ essential partition $P_{n}=\left\{e_{1}^{n}, \ldots, e_{r(n)}^{n}\right\}$ of $H$ with respect to $f$ and a sequence of partitions of unity $\left\{\phi_{m 1}^{n}, \ldots, \phi_{m r(n)}^{n}\right\}$ such that, for $i=1,2, \ldots, r(n), X_{e_{i}^{n} n}^{\prime \prime} n \sim\left\{\phi_{m i}^{n}\right\}$, and $p\left(f-\sum_{i=1}^{r(n)} \phi_{m i}^{n} \cdot x_{i}^{n}\right) \leqq 2 \varepsilon_{n}$ for any choice of the points $x_{i}^{n} \in f\left(e_{i}^{n}\right)$, and for all $m$, $\chi_{e_{i}^{n}}^{\prime \prime} \sim\left\{\phi_{m i}^{n}\right\}$ so $J^{\prime \prime}\left(\chi_{e_{i}^{n}}^{\prime \prime}\right) \sim\left\{J^{\prime \prime}\left(\phi_{m i}^{n}\right)\right\}$, but since $\phi_{m i}^{n} \in C(H, E), J^{\prime \prime}\left(\phi_{m i}^{n}\right)=J\left(\phi_{m k}^{n}\right)$. Then $K\left(e_{i}^{n}\right) \cdot x_{i}^{n} \sim\left\{J\left(\phi_{m i}^{n}\right) x_{i}^{n}\right\}$ and $J\left(\phi_{m i}^{n}\right) \cdot x_{i}^{n}=T\left(\phi_{m i}^{n} \cdot x_{i}\right)$. Thus

$$
\begin{aligned}
q^{+}\left(T(f)-\sum_{i=1}^{r(n)} K\left(e_{i}^{n}\right) \cdot x_{i}^{n}\right) & =\sup _{y^{\prime} \in B_{q}^{O}} \lim _{m}\left|\left\langle T(f)-\sum_{i=1}^{r(n)} T \phi_{m i}^{n} \cdot x_{i}^{n}, y^{\prime}\right\rangle\right| \\
& =\sup _{y^{\prime} \in B_{q}^{O}} \lim _{m}\left|\left\langle T\left(f-\sum_{i=1}^{r(n)} \phi_{m i}^{n} \cdot x_{i}^{m}\right), y^{\prime}\right\rangle\right|
\end{aligned}
$$


But $q T(h) \leqq|T|_{p-q} p(h)$ for every $h \in C(H, E)$. But

$$
q T(h)=\sup _{y^{\prime} \in B_{q}^{O}}\left|\left\langle T(h), y^{\prime}\right\rangle\right|,
$$

hence if $y^{\prime} \in B_{q^{\prime}}^{O},|\langle T(h), y\rangle| \leqq q T(h) \leqq|T|_{p-q} p(h)$. Hence if $y^{\prime} \in B_{q^{\prime}}^{O}$,

$$
\left|\left\langle T\left(f-\sum_{i=1}^{r(n)} \phi_{m i}^{n} \cdot x_{i}^{n}\right), y^{\prime}\right\rangle\right| \leqq|T|_{p-q}\left(f-\sum_{i=1}^{r(n)} \phi_{m i}^{n} \cdot x_{i}^{n}\right) \leqq|T|_{p-q} 2 \varepsilon_{n}
$$

for every $m$. Thus

$$
\lim _{m}\left|\left\langle T\left(f-\sum_{i=1}^{r(n)} \phi_{m i}^{n} \cdot x_{i}^{n}\right), y^{\prime}\right\rangle\right| \leqq|T|_{p-q} 2 \varepsilon_{n}
$$

for every $y^{\prime} \in B_{q}^{O}$. Hence

$$
q^{+}\left(T(f)-\sum_{i=1}^{r(n)} K\left(e_{i}^{n}\right) \cdot x_{i}^{n}\right) \leqq|T|_{p-q} 2 \varepsilon_{n} .
$$

Suppose $d>0$ is given. Choose $n$ such that $4 \varepsilon_{n}|T|_{p-q}<d$. Let $\delta\left(q^{+}, d, p\right)=\varepsilon_{n}$. Suppose $0<\varepsilon<\delta\left(q^{+}, d, p\right)$. If $P=\left\{e_{1}, e_{2}, \ldots, e_{k}\right\}$ is any $\varepsilon-p$ essential partition of $H$ with respect to $f$. It follows that $P$ is an $\varepsilon_{n}-p$ essential partition of $H$ with respect to $f$ since $\varepsilon \leqq \varepsilon_{n}$. Suppose $x_{i} \in e_{i}$, then

$$
\begin{aligned}
q^{+}\left(T(f)-\sum_{i=1}^{k} K\left(e_{i}\right) \cdot x_{i}\right) \leqq & q^{+}\left(T(f)-\sum_{i=1}^{r(n)} K\left(e_{i}^{n}\right) \cdot x_{i}^{n}\right) \\
& +q^{+}\left(\sum_{i=1}^{r(n)} K\left(e_{i}^{n}\right) \cdot x_{i}^{n}-\sum_{i=1}^{k} K\left(e_{i}\right) \cdot x_{i}\right) \\
\leqq & |T|_{p-q} 2 \varepsilon_{n}+|T|_{p-q} 2 \varepsilon_{n},
\end{aligned}
$$

where this last inequality follows from Proposition 3.2. Thus

$$
q^{+}\left(T(f)-\sum_{i=1}^{k} K\left(e_{i}\right) \cdot x_{i}\right)<d .
$$

Proposition 4.1. If $T$ is a continuous linear transformation from $C(H, E)$ into $F$, then there exists a set function $K$ from $R$ into $\Gamma\left(E, F^{+}\right)$such that $\int d K f$ exists for all $f \in C(H, E)$, and $T(f)=\int d K f$. Further, $K$ is of bounded $\left(p, q^{+}\right)$variation, and if $T$ is $p-q$ related then $V(K)_{p-q^{+}}=|T|_{p-q}$.

Proof. The set function $K$ is defined in Definition 2.3. Given $q^{+}$and $d>0$, we know by Lemma 4.1, that there exists a continuous seminorm $p$ on $E$ and a $\delta\left(q^{+}, d, p\right)>0$ such that if $P=\left\{e_{1}, \ldots, e_{k}\right\}$ is any $\varepsilon-p$ essential partition of $H$ with respect to $f$, where $0<\varepsilon \leqq \delta\left(q^{+}, d, p\right)$, then

$$
q^{+}\left(T(f)-\left(T(f)-\sum_{i=1}^{k} K\left(e_{i}\right) \cdot x_{i}\right)\right) \leqq d
$$


for any choice of $x_{i} \in f\left(e_{i}\right)$. Now let $P_{0}=\left\{E_{1}, \ldots, E_{m}\right\}$ be a $\delta\left(q^{+}, d, p\right)-p$ essential partition of $H$ with respect to $f$. (There is one by Lemma 3.2.) If $P=\left\{e_{i}, \ldots, e_{k}\right\}$ is any refinement of $P_{0}$ which is an essential partition, then $P$ is also a $\delta\left(q^{+}, d, p\right)-p$ essential partition of $H$. Hence $q^{+}\left(T(f)-\sum_{i=1}^{k} K\left(e_{i}\right) \cdot x_{i}\right)<d$ for any choice of $x_{i} \in f\left(e_{i}\right)$. Thus $\int d K f$ exists and $T(f)=\int d K f$. Now suppose $T$ is $p-q$ related, then $V(K)_{p-q^{+}} \leqq|T|_{p-q}$ by Proposition 3.1. But

$$
q T(f)=q \int d K f=q^{+}\left(\int d K f\right) \leqq V(K)_{p-q^{+}} p(f)
$$

since

$$
q^{+}\left(\sum K\left(e_{i}\right) \cdot x_{i}\right) \leqq V(K)_{p-q^{+}} \max p\left(x_{i}\right) \leqq V(K)_{p-q^{+}} p(f)
$$

for any choice of $x_{i} \in f\left(e_{i}\right)$ and any essential partition $P=\left\{e_{i}, \ldots, e_{n}\right\}$. Thus $|T|_{p-q}=V(K)_{p-q^{+}}$.

Another question one might ask is, when does a set function which is of bounded $\left(p, q^{+}\right)$variation generate a continuous linear transformation of $C(H, E)$ into $F$ ? One could perhaps add conditions on the set function $K$ or add conditions on the space $F$ to give various answers to this question. Toward this goal we prove

Proposition 4.2. If $K$ is an additive set function from $R$ into $\Gamma(E, F)$ and $K$ is of bounded $(p, q)$ variation, and if $F$ is quasi-complete, i.e., a space in which every closed and bounded set is complete, then $\int d K f$ exists for every $f \in C(H, E)$, and $T(f)=\int d K f$ defines a continuous linear transformation from $C(H, E)$ into $F$.

Proof. Let $A\left(P_{0}\right)=\left\{\sum_{i=1}^{n} K\left(e_{i}\right) \cdot x_{i}\right.$, where $x_{i} \in f\left(e_{i}\right), P=\left\{e_{1}, \ldots, e_{n}\right\}$, and $P$ is any essential partition which is a refinement of the essential partition $\left.P_{0}\right\}$. If $P_{1}$ and $P_{2}$ are two essential partitions then there exists an essential partition $P_{3}$ which is a refinement of both $P_{1}$ and $P_{2}$. (See the first part of the proof of Proposition 3.2.) We see that $A\left(P_{3}\right) \leqq A\left(P_{1}\right) \cap A\left(P_{2}\right)$. Thus the collection $\left\{A\left(P_{4}\right)\right\}$ forms a filter base, see $[4$, p. 56]. It is a Cauchy filter base, for if $\varepsilon>0$ and $p$, a continuous seminorm on $E$, are given, then by Lemma 3.2 there exists an $\varepsilon-p$ essential partition $P_{0}$ of $H$ with respect to $f$. If $P$ is any refinement of $P_{0}, P$ is an $\varepsilon-p$ essential partition of $H$. Considering the proof of Proposition 3.2, we note that if $K$ is of bounded $(p-q)$ variation and $K(\Phi)=0$, which follows from the additivity of $K$, then $A\left(P_{0}\right)$ is of $p$ diameter $\leqq V(K)_{p-q}(2 \varepsilon)$. Thus $\{A(P)\}$ is a Cauchy filter base. Also if we consider $A=\bigcup A(P)$, where the union is over the essential partitions $P$ of $H$, then $A$ is bounded. For

$$
q\left(\sum_{i=1}^{n} K\left(e_{i}\right) \cdot x_{i}\right) \leqq V(K)_{p-q} \max p\left(x_{i}\right) \leqq V(K)_{p-q} p(f),
$$

where $x_{i} \in f\left(e_{i}\right)$ and $p=\left\{e_{1}, \ldots, e_{n}\right\}$ is an essential partition of $H$. Thus $A$ is bounded. But a quasi-complete space is defined to be a space in which closed bounded sets are complete. Thus $\int d K f$ exists. Here we use the fact that $F$ is Hausdorff to assume the uniqueness of the limit. The linearity follows trivially. $T(f)=\int d K f$ is continuous, for from the last inequality above we have $q T(f)=q\left(\int d K f\right) \leqq V(K)_{p-q} p(f)$. 
The converse of this theorem is false. See [6] where an example is given where $E$ and $F$ are Banach spaces and any such $K$ has values in $L\left(E, F^{+}\right)$but not in $L(E, F)$.

PROPOSITION 4.3. If $F$ is semireflexive, then $T$ is a continuous linear transformation of $C(H, E)$ into $F$ if and only if there exists an additive set function $K$ from $R$ into $\Gamma(E, F)$ such that $K$ is of bounded $(p, q)$ variation and $T(f)=\int d K f$.

Proof. A semireflexive space is a space such that $F^{\prime \prime}=F$ (see $[4$, p. 72]). It is known [2, Theorem 20.2, p. 190] that a semireflexive space is quasi-complete. Thus if $K$ is an additive set function from $R$ into $\Gamma(E, F)$ which is of bounded $(p-q)$ variation, then by Proposition 4.2, $T(f)=\int d K f$ defines a continuous linear transformation of $C(H, E)$ into $F$. Next suppose $T$ is a continuous linear transformation of $C(H, E)$ into $F$. By Proposition 4.1 there exists an additive set function $K$ from $R$ into $\Gamma\left(E, F^{+}\right)$of bounded $\left(p, q^{+}\right)$variation such that $T(f)=\int d K f$. But $F^{\prime \prime} \supset F^{+} \supset F=F^{\prime \prime}$, hence $F^{+}=F$, and $K$ is an additive set function from $R$ into $\Gamma(E, F) . K$ is of bounded $(p-q)$ variation, since $q$ agrees with $q^{+}$on $F$, and $K$ is of bounded $\left(p-q^{+}\right)$variation.

5. Concerning the ring $R$. One might ask how the ring $R$ is related to $R(S)$, where $S$ is the collection of all compact $G_{\delta}$ 's of $H$ and $R(S)$ is the ring generated by $S$. The answer to this question is given in the following proposition.

Proposition 5.1. $R=R(S)$.

Proof. By straightforward arguments one has $\sigma(S) \supset R \supset R(S)$, where $\sigma(S)$ is the $\sigma$-ring generated by $S$.

Suppose now that $A \in R$. We define the sequence $\Phi_{n}$ of sets by induction. Let $\Phi_{0}=\mathrm{cl}(\mathrm{cl}(A) \cap \mathrm{c}(A))$, where $\mathrm{cl}(B)$ denotes the closure of $B$ and $\mathrm{c}(B)$ denotes the complement of $B$ for any subset $B$ of $H$. Define $\Phi_{n}=\operatorname{cl}\left(\operatorname{cl}\left(\Phi_{n-1} \cap A\right) \cap \mathrm{C}(A)\right)$. Let $\phi_{n}=f_{n}-g_{n}$ where $\phi_{n}, f_{n}$, and $g_{n}$ are sequences of functions satisfying the conditions of Definition 2.2. Let $f=\lim f_{n}$ and $g=\lim g_{n}$. Then, using the fact that $f$ and $g$ are upper-semicontinuous, one can show by induction that $f(x) \geqq n+1$, and $g(x) \geqq n+1$ for all $x \in \Phi_{n}$. We see then that for $A \in R, \Phi_{n}$ is empty for large enough $n$.

We next define the mapping $\Psi$ of the subsets of $H$ into the subsets of $H$ by $\Psi(B)=\mathrm{cl}(B) \cap \mathrm{c}(B)$. By elementary set calculations and by induction, we have $\Psi^{2 n}(A)=\left(\Phi_{n-1} \cap A\right)$. We thus see that if $A$ is in $R, \Psi^{n}(A)$ is empty for large enough $n$.

We now refer to the proof of [1, Theorem D, p. 221], and by imitating this proof, we construct a continuous function $F$ from $H$ onto a compact metric space $H^{\prime}$ with the important property that if $F(x)=F(y)$ and $x \in A$ then $y \in A$. Now using this fact and the identities $\Psi^{2 n}(A)=\operatorname{cl}\left(\Psi^{2 n-1}(A)\right) \cap A$ and $\Psi^{2 n+1}(A)=\operatorname{cl}\left(\Psi^{2 n}(A)\right)$ $\cap \mathrm{c}(A)$, we have by induction $\Psi^{n}(F(A)) \subset F\left(\Psi^{n}(A)\right)$.

Let $B=F(A) . B=\mathrm{cl}(B) \cap \mathrm{c}(\Psi(B))$, and so if $\Psi(B)$ is in $R^{\prime}$, the ring generated by the compact subsets of $H^{\prime}$, then $B$ is in $R^{\prime}$. By replacing $B$ by $\Psi(B)$ in the 
above argument one sees that $\Psi^{\prime}(B) \in R^{\prime}$ if $\Psi^{2}(B) \in R^{\prime}$; and a trivial induction argument shows that if $\Psi^{n}(B) \in R^{\prime}$ for any $n$, then $B \in R^{\prime}$. But this is the case as $\Psi^{n}(B)$ is empty for large enough $n$. Since $H^{\prime}$ is a metric space $R(S)=R^{\prime}$, and for this particular function $F, A=F^{-1}(B)$. But inverse images of elements of $S$ in $H^{\prime}$ are in $S$ in $H$; and the collection of all subsets $C$ of $H^{\prime}$, for which $F^{-1}(C)$ is in $R(S)$ in $H$, is itself a ring; and so $A \in R(S)$ in $H$. Hence $R=R(S)$ in $H$.

Note. We have developed a simple test to determine when a set $A$ is in $R(S)$. If $A \in \sigma(S)$ then $A \in R(S)$ if and only if $\Psi^{n}(A)$ is empty for large enough $n$.

6. Concerning the uniqueness of the set function $K$. For the sake of simplicity we now restrict our attention to the case when our field is the real numbers. Similar type theorems with similar proofs hold in the complex case.

Definition 6.1. If $\lambda$ is a real valued nonnegative additive set function on $R$, then we say $\lambda$ is continuous from above provided that $\lambda(c)=\lim \lambda\left(c_{n}\right)$ whenever $c_{n}$ is a sequence of elements of $S$ such that $c_{n+1} \subset c_{n}$ and $\bigcap c_{n}=c$.

Definition 6.2. If $W$ is an additive set function from $R$ into $\Gamma\left(E, F^{+}\right)$of bounded $p-q^{+}$variation, then we say $W$ is weakly continuous from above provided that for each $x \in E$ and $y^{\prime} \in F^{\prime},|\lambda|$ is continuous from above, where $|\lambda|$ is the variation of the set function $\lambda$ defined by $\lambda(e)=\left\langle W(e) \cdot x, y^{\prime}\right\rangle$. We define $|\lambda|(e)=\sum\left|\lambda\left(e \cap e_{i}\right)\right|$ where the supremum is taken over all essential partitions $\left\{e_{1}, e_{2}, \ldots, e_{n}\right\}$ of $H$. One can show $|\lambda|$ is an additive set function on $R$.

Definition 6.3. If $\lambda$ is an additive real valued set function on $R$, then we say $\lambda$ is sequentially continuous provided that for each $e$ in $R, \lambda(e)=\lim \int d \lambda \phi_{n}$ for any sequence $\phi_{n}$ satisfying the conditions of Definition 2.2.

LEMMA 6.1. If $\lambda$ is an additive real valued set function of bounded variation, then $\lambda$ is sequentially continuous if and only if $|\lambda|$ is continuous from above.

Proof. First let us suppose $|\lambda|$ is continuous from above. $|\lambda|=\lambda_{+}+\lambda_{-}$where $\lambda_{+}=(|\lambda|-\lambda) / 2 . \lambda_{+}$and $\lambda_{-}$are nonnegative additive set functions on $R$, and

$$
|\lambda|\left(c_{n}\right)-|\lambda|(c) \geqq \lambda_{+}\left(c_{n}\right)-\lambda_{+}(c) \geqq 0,
$$

so $\lambda_{+}$and $\lambda_{-}$are continuous from above.

We will now show $\lambda_{+}$is sequentially continuous. Let us suppose $c \in S$. By [1, Theorem 6, p. 217] there exists a nonnegative continuous function $f$ such that $c=\{x \mid f(x)=0\}$. Let $c_{n}=\{x \mid f(x) \leqq 1 / n\}, O_{n}=\{x \mid f(x)<1 / n\}$. Now pick a sequence of continuous functions $f_{n}$ such that $0 \leqq f_{n} \leqq 1 ; f_{n}=1$ on $c_{n} ; f_{n}$ is zero outside of $O_{n-1}$ and $f_{n}$ is monotonically nonincreasing. $\lambda_{+}(c) \leqq \int d \lambda_{+} f_{n} \leqq \lambda\left(c_{n-1}\right)$, so $\lambda_{+}(c)$ $=\lim \int d \lambda_{+} f_{n}$, and since we know the limit is independent of $\phi_{n}$, we have $\lambda_{+}(c)=\lim \int d \lambda_{+} \phi_{n}$ for any sequence $\phi_{n}$ satisfying the conditions of Definition 2.2. To see why $\lambda_{+}$is sequentially continuous for all of $R$, recall that $R=R(S)$ and note that by [1, Theorem F, p. 223] every element of $R(S)$ is the finite disjoint union of proper differences of elements of $S$. By a similar argument, $\lambda_{-}$is sequentially continuous, and since $\lambda=\lambda_{+}-\lambda_{-}$, we see $\lambda$ is sequentially continuous. 
Next let us suppose $\lambda$ is sequentially continuous and of bounded variation. Let $h(f)=\int d \lambda f$, then $h \in(c(H))^{\prime}$; and it is well known that there exist elements $h_{1}$ and $h_{2}$ of $(c(H))^{\prime}$ for which $h=h_{1}-h_{2}$, and $h_{i}(f) \geqq 0$ whenever $f \geqq 0$ for $i=1,2$. But by Proposition 4.1, we know there exist additive set functions $\lambda_{1}$ and $\lambda_{2}$ that are sequentially continuous for which $h_{i}(f)=\int d \lambda_{i} f$ for all $f \in(H)$ and $i=1,2$, and since $\lambda, \lambda_{1}$, and $\lambda_{2}$ are all sequentially continuous we have $\lambda=\lambda_{1}-\lambda_{2}$. Also by using [1, Theorem F, p. 223] one can show that $\lambda_{1}$ and $\lambda_{2}$ are nonnegative on $R$.

We will now show $\lambda_{1}$ is continuous from above. Suppose $c=\bigcap c_{n}$ where $c_{n}$ is a monotone nonincreasing sequence in $S$, i.e. $c_{n+1} \subset c_{n}$. Pick a sequence of open sets $O_{n i}$ such that $O_{n i}$ is monotone nonincreasing in $n$ and in $i$ and such that $\bigcap_{i} O_{n i}=c_{n}$. Now pick a sequence of continuous functions $f_{n}$ for which $0 \leqq f_{n} \leqq 1, f_{n}=1$ on $c_{n}$ and $f_{n}$ is zero outside of $O_{n n}$ and such that $f_{n}$ is monotonically nonincreasing. $\lambda_{1}(c) \leqq \lambda_{1}\left(c_{n}\right) \leqq \int d \lambda_{1} f_{n}$, so $\lambda_{1}(c)=\lim \lambda_{1}\left(c_{n}\right)$. A similar argument holds for $\lambda$.

$$
0 \leqq|\lambda|\left(c_{n}\right)-|\lambda|\left(c_{n}\right)=|\lambda|\left(c_{n}-c\right) \leqq \lambda_{1}\left(c_{n}\right)-\lambda_{1}(c)+\lambda_{2}\left(c_{n}\right)-\lambda_{2}(c),
$$

so $|\lambda|$ is continuous from above.

Proposition 6.1. There is one and only one additive set function $K$ of bounded $p-q^{+}$variation on $R$ that is weakly continuous from above for which $T(f)=\int d K f$ for all $f$ in $C(H, E)$.

Proof. Consider the set function $K$ defined in Definition 2.3.

$$
\begin{aligned}
\lambda(e) & =\left\langle K(e) \cdot x, y^{\prime}\right\rangle=\lim \left\langle T\left(\phi_{n} \cdot x\right), y^{\prime}\right\rangle \\
& =\lim \left\langle\int d K \phi_{n} x, y^{\prime}\right\rangle=\lim \int d \lambda \phi_{n}
\end{aligned}
$$

for any sequence $\phi_{n}$ satisfying the conditions of Definition 2.2, thus $\lambda$ is sequentially continuous and, by Lemma $6.1, K$ is weakly additive from above.

Suppose $K_{1}$ is any other such set function, then $\lambda_{1}(c)=\left\langle K_{1}(c) \cdot x, y^{\prime}\right\rangle$ is sequentially continuous and so if $\phi_{n}$ is a sequence satisfying the conditions of Definition 2.2,

$$
\begin{aligned}
\left\langle K_{1}(c) \cdot x, y^{\prime}\right\rangle & =\lambda_{1}(c)=\lim \int d \lambda \phi_{n}=\lim \left\langle\int d K_{1}\left(\phi_{n} \cdot x\right), y^{\prime}\right\rangle \\
& =\lim \left\langle T\left(\phi_{n} \cdot x\right), y^{\prime}\right\rangle=\left\langle K(c) \cdot x, y^{\prime}\right\rangle .
\end{aligned}
$$

But this is true for all $x$ in $E$, all $c$ in $R$, and all $y^{\prime}$ in $F^{\prime}$, so $K_{1}=K$.

\section{REFERENCES}

1. P. R. Halmos, Measure theory, Univ. Ser. Higher Math., Van Nostrand, New York, 1950.

2. J. L. Kelley, I. Namioka, et al., Linear topological vector spaces, Univ. Ser. Higher Math., Van Nostrand, New York, 1963.

3. F. Riesz, Sur les opérations fonctionelles linéaires, C. R. Acad. Sci. Paris 149 (1909), 974-977. 
4. A. P. Robertson and W. J. Robertson, Topological vector spaces, Cambridge Tracts in Math. and Math. Physics, No. 53. Cambridge Univ. Press, Cambridge, 1964.

5. K. Swong, A representation theory of continuous linear maps, Math. Ann. 155 (1964), 270-291.

6. D. H. Tucker, A representation theorem for a continuous linear transformation on a space of continuous functions, Proc. Amer. Math. Soc. 16 (1965), 946-953.

7. D. J. Uherka, A Riesz representation theorem, Ph.D. Thesis, University of Utah, Salt Lake City, 1964.

\section{UNIVERSITY OF COLORADO,}

BOULDER, COLORADO 